\title{
n-NORMED HYPERVECTOR SPACES
}

\section{SEDGHI, O. R. DEHGHAN and M. NOROUZI}

Department of Mathematics

Faculty of Basic Sciences

University of Bojnord

Bojnord

Iran

e-mails: mozhgan-sedghi@yahoo.com

dehghan@ub.ac.ir

m.norouzi@ub.ac.ir

\begin{abstract}
In this paper, we introduce the concept of $n$-norm on hypervector spaces and investigate how we can obtain $n$-normed hypervector spaces from normed hypervector spaces. By defining $n$-homomorphisms on $n$-normed hypervector spaces and a suitable norm for them, we investigate some of their properties. Moreover, the notion of quasi- $n$-normed subhyperspaces is introduced and some results about them are obtained.
\end{abstract}

\section{Introduction}

The notion of a hypergroup was introduced by Marty in 1934 [7]. Since then many researchers have worked on hyperalgebraic structures and developed this theory (for more information, see $[3,4,5,13]$ ). In 1990, Tallini [10] introduced the notion of hypervector spaces and studied 
basic properties of them. In 2008, Ameri and Dehghan [2] investigated some topics in such spaces. Also, some results in this context can be seen in $[1,12]$ and $[14]$.

The theory of 2-normed and $n$-normed spaces was developed by Gähler [6] and Misiak [8], respectively. Recently, Raja [9] studied some properties of 2-norms on hypervector spaces.

In this paper, we generalize the notions of norm, homomorphism and quasi-norm in hypervector spaces and obtain some interesting results.

\section{Preliminaries}

In this section, we present some definitions and simple properties of normed hypervector spaces, that we shall use in later.

Definition 2.1 ([10]). Let $F$ be a field and $(V,+)$ be an abelian group. We define a hypervector space over $F$ to be the quadruple $(V,+, \circ, F)$, where "o" (external hyperoperation) is a mapping

$$
\circ: F \times V \rightarrow P^{*}(V)
$$

such that for all $a, b \in F$ and $x, y \in V$ the following conditions hold $\left(P^{*}(V)\right.$ denotes the set of all nonempty subsets of $\left.V\right)$ :

$\left(\mathrm{H}_{1}\right) a \circ(x+y) \subseteq a \circ x+a \circ y$, where $a \circ x+a \circ y=\{p+q: p \in a \circ x$, $q \in a \circ y\}$

$\left(\mathrm{H}_{2}\right)(a+b) \circ x \subseteq a \circ x+b \circ x ;$

$\left(\mathrm{H}_{3}\right) a \circ(b \circ x)=(a b) \circ x$, where $a \circ(b \circ x)=\bigcup_{y \in b \circ x} a \circ y$;

$\left(\mathrm{H}_{4}\right) a \circ(-x)=(-a) \circ x=-(a \circ x)$;

$\left(\mathrm{H}_{5}\right) x \in 1 \circ x$.

We say that $(V,+, \circ, F)$ is strongly left distributive, if $(a+b) \circ x=$ $a \circ x+b \circ x$. 
A nonempty subset $W$ of hypervector space $(V,+, \circ, F)$ is called a subhyperspace of $V$ if $W-W \subseteq W$ and $a \circ W \subseteq W$, for all $a \in F$.

Example 2.2 ([9]). In classical vector space $\left(\mathbb{R}^{n},+, ., \mathbb{R}\right)$, we define

$$
\left\{\begin{array}{l}
\circ: \mathbb{R} \times \mathbb{R}^{n} \rightarrow P^{*}\left(\mathbb{R}^{n}\right), \\
a \circ\left(x_{1}, \ldots, x_{n}\right)=\left\{\left(\operatorname{tax}_{1}, \ldots, \operatorname{tax}_{n}\right): 0 \leq t \leq 1\right\} .
\end{array}\right.
$$

Then $V=\left(\mathbb{R}^{n},+, \circ, \mathbb{R}\right)$ is a hypervector space.

Definition 2.3 ([2]). A subset $S$ of $V$ is called linearly independent if for every vectors $v_{1}, v_{2}, \ldots, v_{n}$ in $S$, and $c_{1}, \ldots, c_{n} \in K, \underline{0}=0_{V} \in c_{1} \circ v_{1}$ $+\cdots+c_{n} \circ v_{n}$, implies that $c_{1}=c_{2}=\cdots=c_{n}=0$. A subset $S$ of $V$ is called linearly dependent, if it is not linearly independent. A basis for $V$ is a linearly independent subset of $V$ such that span $V$.

Note that some hypervector spaces $V$ may not have any collection of linearly independent vectors. Such hypervector space is called independentless.

Definition 2.4 ([11]). Let $(V,+, \circ, F)$ be a hypervector space. Then a mapping $\|\cdot\|: V \rightarrow \mathbb{R}$ is called a norm on $V$, if for all $x, y \in V$ and $a \in F$, the followings hold:

(N1) $\|x\|=0$ if and only if $x=0$;

(N2) $\|x+y\| \leq\|x\|+\|y\| ;$

(N3) $\sup \|a \circ x\|=|a|\|x\|$;

where $\sup \|a \circ x\|=\sup \{\|t\|: t \in a \circ x\}$. In this case, $(V,+, \circ, F,\|\cdot\|)$ is called a normed hypervector space.

Definition 2.5 ([11]). Let $\left(V,{ }_{1},{ }^{\circ}, F\right)$ and $\left(W,+_{2},{ }_{2}, F\right)$ be two hypervector spaces. Then a mapping $T: V \rightarrow W$ is called good linear transformation iff $T(x+1 y)=T(x)+{ }_{2} T(y)$ and $T\left(a \circ_{1} x\right)=a \circ_{2} T(x)$, for all $a \in F$ and $x, y \in V$. 


\section{3. $n$-Norms on Hypervector Spaces}

In this section, $n$-norm on hypervector spaces is defined as a generalization of $n$-norm on vector spaces. The connection between $n$-normed hypervector spaces and normed hypervector spaces are investigated.

Note that, for abbreviation, some times a sequence $x_{i}, x_{i+1}, \ldots, x_{j}$ is denoted by $x_{i}^{j}$.

Definition 3.1. Let $(V,+, \circ, \mathbb{R})$ be a real hypervector space such that $\operatorname{dim}(V) \geq n$. Then a mapping $\|, \ldots,\|:. V^{n} \rightarrow \mathbb{R}$ is called an $n$-norm on $V$, if for all $x_{1}, \ldots, x_{n}, y, z \in V$ and $a \in \mathbb{R}$ the following conditions are satisfied:

(nN1) $\left\|x_{1}, \ldots, x_{n}\right\|=0 \Leftrightarrow\left\{x_{1}, \ldots, x_{n}\right\}$ is linearly dependent;

(nN2) $\left\|x_{1}, \ldots, x_{n}\right\|=\left\|x_{\sigma(1)}, \ldots, x_{\sigma(n)}\right\|$, for all $\sigma \in \mathbb{S}_{n}$;

(nN3) $\left\|x_{1}, \ldots, x_{n-1}, y+z\right\| \leq\left\|x_{1}, \ldots, x_{n-1}, y\right\|+\left\|x_{1}, \ldots, x_{n-1}, z\right\|$;

$(\mathrm{nN} 4) \sup \left\|x_{1}, \ldots, x_{n-1}, a \circ x_{n}\right\|=|a|\left\|x_{1}, \ldots, x_{n}\right\|$.

In this case, $(V,+, \circ, \mathbb{R},\|, \ldots,\|$.$) is called an n$-normed hypervector space.

Example 3.2. Consider the hypervector space $\left(\mathbb{R}^{n},+, \circ, \mathbb{R}\right)$ in Example 2.2. For all $X_{1}^{n} \in \mathbb{R}^{n}$, let $\left\|X_{1}, \ldots, X_{n}\right\|=\prod_{i=1}^{n} s_{i}$, where $s_{i}$ is the distance of $X_{i}$ from the origin in $\mathbb{R}^{n}$, for any $1 \leq i \leq n$. Then $\left(\mathbb{R}^{n},+\right.$, 。, $\mathbb{R},\|, \ldots,\|$.$) is an n$-normed hypervector space.

It is clear that every $n$-normed vector space can be extended to an $n$-normed hypervector space by a trivial external hyperoperation. 
The next theorem presents a method to construct an $n$-normed hypervector space from a normed hypervector space under specific conditions.

Theorem 3.3. Suppose that $(V,+, \circ, \mathbb{R})$ is a normed strongly left distributive hypervector space with $\operatorname{dim}(V) \geq n$, such that the following conditions are satisfied:

(1) $|0 \circ x|=1$, for all $x \in V$;

(2) $\underline{0} \notin a \circ x$, for all nonzero scalars $a \in \mathbb{R}$ and nonzero vectors $x \in V$;

(3) $\underline{0} \notin A, B$, implies that $\underline{0} \notin A+B$, for all subsets $A, B \subseteq V$.

Then $(V,+, \circ, \mathbb{R})$ is n-normed.

Proof. For any $x_{1}^{n} \in V$, define

$$
\left\|x_{1}, \ldots, x_{n}\right\|=\left\|x_{1}\right\| \cdots\left\|x_{n}\right\|
$$

Then

nN1. If $\left\|x_{1}, \ldots, x_{n}\right\|=0$, then $\left\|x_{1}\right\| \cdots\left\|x_{n}\right\|=0$. Thus $\left\|x_{i}\right\|=0$, for some $1 \leq i \leq n$. By (N1), it follows that $x_{i}=\underline{0}$. Hence, $\underline{0}=\underline{0}+\cdots+\underline{0}+x_{i}+\underline{0}+\cdots+\underline{0} \in 0 \circ x_{1}+\cdots+0 \circ x_{i-1}+1 \circ x_{i}+0 \circ x_{i+1}$

$$
+\cdots+0 \circ x_{n}
$$

Therefore, $\left\{x_{1}, \ldots, x_{n}\right\}$ is linearly dependent. Conversely, let $\left\|x_{1}, \ldots, x_{n}\right\| \neq 0$. Then $\left\|x_{i}\right\| \neq 0$, for all $1 \leq i \leq n$. So $x_{i} \neq \underline{0}$, for all $1 \leq i \leq n$. Now if $\left\{x_{1}, \ldots, x_{n}\right\}$ is linearly dependent, then $\underline{0} \in a_{1} \circ x_{1}+\cdots+a_{n} \circ x_{n}$, such that $a_{j} \neq 0$, for some $1 \leq j \leq n$. By the conditions (1), (2), and (3), it follows that, $\underline{0} \notin a_{1} \circ x_{1}+\cdots+a_{n} \circ x_{n}$, which is a contradiction. Hence, $\left\{x_{1}, \ldots, x_{n}\right\}$ is linearly independent. 
nN2. Since multiplication of real numbers is commutative, for all $\sigma \in \mathbb{S}_{n}$, we have:

$$
\begin{aligned}
\left\|x_{1}, \ldots, x_{n}\right\| & =\left\|x_{1}\right\| \cdots\left\|x_{n}\right\| \\
& =\left\|x_{\sigma(1)}\right\| \cdots\left\|x_{\sigma(n)}\right\| \\
& =\left\|x_{\sigma(1)}, \ldots, x_{\sigma(n)}\right\| .
\end{aligned}
$$

nN3. For all $x_{1}, \ldots, x_{n-1}, y, z \in V$, we have:

$$
\begin{aligned}
\left\|x_{1}, \ldots, x_{n-1}, y+z\right\| & =\left\|x_{1}\right\| \cdots\left\|x_{n-1}\right\|\|y+z\| \\
& \leq\left\|x_{1}\right\| \cdots\left\|x_{n-1}\right\|(\|y\|+\|z\|) \\
& =\left\|x_{1}\right\| \cdots\left\|x_{n-1}\right\|\|y\|+\left\|x_{1}\right\| \cdots\left\|x_{n-1}\right\|\|z\| \\
& =\left\|x_{1}, \ldots, x_{n-1}, y\right\|+\left\|x_{1}, \ldots, x_{n-1}, z\right\| .
\end{aligned}
$$

nN4. Since $V$ is a normed hypervector space, then for all $x_{1}, \ldots, x_{n} \in V$ and $a \in \mathbb{R}$ it follows that:

$$
\begin{aligned}
\sup \left\|x_{1}, \ldots, x_{n-1}, a \circ x_{n}\right\| & =\sup _{t \in a \circ x_{i}}\left\|x_{1}, \ldots, x_{n-1}, t\right\| \\
& =\sup _{t \in a \circ x_{i}}\left\|x_{1}\right\| \cdots\left\|x_{n-1}\right\|\|t\| \\
& =\left\|x_{1}\right\| \cdots\left\|x_{n-1}\right\||a|\left\|x_{n}\right\| \\
& =|a|\left\|x_{1}, \ldots, x_{n}\right\| .
\end{aligned}
$$

Therefore, $(V,+, \circ, \mathbb{R})$ is an $n$-normed hypervector space.

\section{4. -Homomorphisms on Hypervector Spaces}

In this section, the concept of $n$-homomorphism on hypervector spaces is introduced and it is shown that the set of all bounded $n$ homomorphism on normed hypervector spaces is a normed hypervector space, under a suitable norm. Also some results about them is obtained. 
We recall that if $(V,+, \circ, \mathbb{R})$ is a real hypervector space, then $\left(V^{n}, \oplus, \odot, \mathbb{R}\right)$ is also a hypervector space with

$$
\begin{gathered}
\left(x_{1}^{n}\right) \oplus\left(y_{1}^{n}\right)=\left(x_{1}+y_{1}, \ldots, x_{n}+y_{n}\right), \\
\alpha \odot\left(x_{1}^{n}\right)=\left(\alpha \circ x_{1}, \ldots, \alpha \circ x_{n}\right)=\bigcup_{\substack{t_{i} \in \alpha \circ x_{i} \\
1 \leq i \leq n}}\left(t_{i}^{n}\right),
\end{gathered}
$$

for all $\left(x_{1}^{n}\right),\left(y_{1}^{n}\right) \in V^{n}, \alpha \in \mathbb{R}$.

Definition 4.1. Assume that $\left(V,{ }_{1},{ }^{\circ} 1, \mathbb{R}\right)$ is an $n$-normed hypervector space and $\left(W,+_{2},{ }_{2}, \mathbb{R}\right)$ is a normed hypervector space. The mapping $F: V^{n} \rightarrow W$ is called an $n$-homomorphism from $V^{n}$ to $W$, if for all $a, x_{1}^{n} \in V, \alpha \in \mathbb{R}$ and $1 \leq i \leq n$, we have

$$
\begin{gathered}
F\left(x_{1}^{i-1}, x_{i}+_{1} a, x_{i+1}^{n}\right)=F\left(x_{1}^{n}\right)+_{2} F\left(x_{1}^{i-1}, a, x_{i+1}^{n}\right), \\
F\left(x_{1}^{i-1}, \alpha \circ_{1} x_{i}, x_{i+1}^{n}\right)=\alpha \circ_{2} F\left(x_{1}^{n}\right) .
\end{gathered}
$$

We say that an $n$-homomorphism $F$ is bounded, if there exists $M>0$ such that

$$
\left\|F\left(x_{1}^{n}\right)\right\| \leq M\left\|x_{1}, \ldots, x_{n}\right\|, \forall x_{1}^{n} \in V
$$

The set of all bounded $n$-homomorphisms from $V^{n}$ to $W$ is denoted by $L_{n}\left(V^{n}, W\right)$.

Proposition 4.2. Let $\left(V,+_{1},{ }^{\circ}, \mathbb{R}\right)$ be an n-normed hypervector space and $\left(W,+_{2},{ }_{2}, \mathbb{R}\right)$ be a normed strongly right distributive hypervector space. For all $F, G \in L_{n}\left(V^{n}, W\right), \alpha \in \mathbb{R}$ and $x_{1}^{n} \in V$, suppose that

$$
\begin{aligned}
(F \oplus G)\left(x_{1}^{n}\right) & =F\left(x_{1}^{n}\right)+_{2} G\left(x_{1}^{n}\right), \\
\alpha \circ F & =\left\{H \in L_{n}\left(V^{n}, W\right) \mid H\left(x_{1}^{n}\right) \in \alpha \circ_{2} F\left(x_{1}^{n}\right), \forall x_{1}^{n} \in V\right\} .
\end{aligned}
$$

Then $\left(L_{n}\left(V^{n}, W\right), \oplus, \circ, \mathbb{R}\right)$ is a real hypervector space. 
Proof. For all $a, x_{1}^{n} \in V$, we have

$$
\begin{aligned}
(F \oplus G)\left(x_{1}^{i-1},\right. & \left.a+{ }_{1} x_{i}, x_{i+1}^{n}\right)=F\left(x_{1}^{i-1}, a+{ }_{1} x_{i}, x_{i+1}^{n}\right)+_{2} G\left(x_{1}^{i-1}, a+{ }_{1} x_{i}, x_{i+1}^{n}\right) \\
& =F\left(x_{1}^{i-1}, a, x_{i+1}^{n}\right)+_{2} F\left(x_{1}^{n}\right)+{ }_{2} G\left(x_{1}^{i-1}, a, x_{i+1}^{n}\right)+_{2} G\left(x_{1}^{n}\right) \\
& =F\left(x_{1}^{i-1}, a, x_{i+1}^{n}\right)+{ }_{2} G\left(x_{1}^{i-1}, a, x_{i+1}^{n}\right)+{ }_{2} F\left(x_{1}^{n}\right)+{ }_{2} G\left(x_{1}^{n}\right) \\
& =(F \oplus G)\left(x_{1}^{i-1}, a, x_{i+1}^{n}\right)+{ }_{2}(F \oplus G)\left(x_{1}^{n}\right) .
\end{aligned}
$$

Also, for all $\alpha \in \mathbb{R}$

$$
\begin{aligned}
(F \oplus G)\left(x_{1}^{i-1}, \alpha \circ_{1} x_{i}, x_{i+1}^{n}\right) & =F\left(x_{1}^{i-1}, \alpha \circ_{1} x_{i}, x_{i+1}^{n}\right)+_{2} G\left(x_{1}^{i-1}, \alpha \circ_{1} x_{i}, x_{i+1}^{n}\right) \\
& =\alpha \circ_{2} F\left(x_{1}^{n}\right)+_{2} \alpha \circ_{2} G\left(x_{1}^{n}\right) \\
& =\alpha \circ_{2}(F \oplus G)\left(x_{1}^{n}\right) .
\end{aligned}
$$

Hence, " $\oplus$ " is well-defined and clearly "o" is also. Moreover, it is easy to see that $\left(L_{n}\left(V^{n}, W\right), \oplus\right)$ is an abelian group which the zero is zero homomorphism and $-F\left(x_{1}^{n}\right)$ is the inverse of any homomorphism $F \in\left(L_{n}\left(V^{n}, W\right), \oplus\right)$. Furthermore, for all $\quad \alpha, \beta \in \mathbb{R} \quad$ and $F, G \in L_{n}\left(V^{n}, W\right)$, we have

$$
\begin{aligned}
\alpha \circ & (F \oplus G) \\
= & \left\{T \in L_{n}\left(V^{n}, W\right): T\left(x_{1}^{n}\right) \in(F+G)\left(x_{1}^{i-1}, \alpha \circ_{1} x_{i}, x_{i+1}^{n}\right), \quad \forall x_{1}^{n} \in V\right\} \\
= & \left\{T \in L\left(V^{n}, W\right): T\left(x_{1}^{n}\right) \in F\left(x_{1}^{i-1}, \alpha \circ_{1} x_{i}, x_{i+1}^{n}\right)+_{2} G\left(x_{1}^{i-1}, \alpha \circ_{1} x_{i}, x_{i+1}^{n}\right), \forall x_{1}^{n} \in V\right\} \\
= & \left\{K \in L\left(V^{n}, W\right): K\left(x_{1}^{n}\right) \in F\left(x_{1}^{i-1}, \alpha \circ_{1} x_{i}, x_{i+1}^{n}\right), \quad \forall x_{1}^{n} \in V\right\} \\
& \oplus\left\{K^{\prime} \in L\left(V^{n}, W\right): K^{\prime}\left(x_{1}^{n}\right) \in G\left(x_{1}^{i-1}, \alpha \circ_{1} x_{i}, x_{i+1}^{n}\right), \quad \forall x_{1}^{n} \in V\right\} \\
= & \alpha \circ F \oplus \alpha \circ G .
\end{aligned}
$$


Similarly, it can be shown that $(\alpha+\beta) \circ F=\alpha \circ F \oplus \beta \circ F$. As well as,

$$
\begin{aligned}
\alpha \circ & (\beta \circ F)=\alpha \circ\left\{H \in L_{n}\left(V^{n}, W\right): H\left(x_{1}^{n}\right) \in \beta \circ_{2} F\left(x_{1}^{n}\right), \quad \forall x_{1}^{n} \in V\right\} \\
= & \bigcup_{H\left(x_{1}^{n}\right) \in \beta_{2} F\left(x_{1}^{n}\right)}(\alpha \circ H) \\
= & \bigcup_{H\left(x_{1}^{n}\right) \in \beta_{2} F\left(x_{1}^{n}\right)}\left\{K \in L_{n}\left(V^{n}, W\right): K\left(x_{1}^{n}\right) \in \alpha \circ_{2} H\left(x_{1}^{n}\right), \quad \forall x_{1}^{n} \in V\right\} \\
= & \left\{K \in L_{n}\left(V^{n}, W\right): K\left(x_{1}^{n}\right) \in \alpha \circ_{2}\left(\beta \circ_{2} F\left(x_{1}^{n}\right)\right), \quad \forall x_{1}^{n} \in V\right\} \\
= & \left\{K \in L_{n}\left(V^{n}, W\right): K\left(x_{1}^{n}\right) \in F\left(x_{1}^{i-1}, \alpha \circ_{1}\left(\beta \circ_{1} x_{i}\right), x_{i+1}^{n}\right), \quad \forall x_{1}^{n} \in V\right\} \\
= & \left\{K \in L_{n}\left(V^{n}, W\right): K\left(x_{1}^{n}\right) \in F\left(x_{1}^{i-1},(\alpha \beta) \circ_{1} x_{i}, x_{i+1}^{n}\right), \quad \forall x_{1}^{n} \in V\right\} \\
= & (\alpha \beta) \circ F .
\end{aligned}
$$

In addition, we can see $-\alpha \circ F=-(\alpha \circ F)$. Also, since for all $x_{1}^{n} \in V$ and $F \in L_{n}\left(V^{n}, W\right)$ we have $F\left(x_{1}^{n}\right) \in W$ and $W$ is a hypervector space, then

$$
F\left(x_{1}^{n}\right) \in 1 \circ_{2} F\left(x_{1}^{n}\right)=F\left(x_{1}^{i-1}, 1 \circ_{1} x_{i}, x_{i+1}^{n}\right) .
$$

As regards $1 \circ F=\left\{T \in L_{n}\left(V^{n}, W\right): T\left(x_{1}^{n}\right) \in F\left(x_{1}^{i-1}, 1 \circ_{1} x_{i}, x_{i+1}^{n}\right), \forall x_{1}^{n} \in V\right\}$, for any $F \in L_{n}\left(V^{n}, W\right), F \in 1 \circ F$. Therefore, $L_{n}\left(V^{n}, W\right)$ is a real hypervector space.

Proposition 4.3. Let $\left(V,+_{1},{ }^{\circ}, \mathbb{R}\right)$ be an n-normed hypervector space and $\left(W,+_{2}, \circ_{2}, \mathbb{R}\right)$ be a normed hypervector space and $F: V^{n} \rightarrow W$ be a bounded n-homomorphism. Define

$$
\|F\|=\inf \left\{M>0: \forall x_{1}^{n} \in V,\left\|F\left(x_{1}^{n}\right)\right\| \leq M\left\|x_{1}, \ldots, x_{n}\right\|\right\} .
$$

Then $\|\cdot\|$ is a norm on $L_{n}\left(V^{n}, W\right)$. 
Proof. If $\|F\|=0$, then $\left\|F\left(x_{1}^{n}\right)\right\|=0$, for all $x_{1}^{n} \in V$. Thus $F\left(x_{1}^{n}\right)=0$, for all $x_{1}^{n} \in V$. Conversely, if $F$ is a zero bounded $n$-homomorphism, then

$$
\|F\|=\sup \left\{\left\|F\left(x_{1}^{n}\right)\right\|: x_{1}^{n} \in V,\left\|x_{1}, \ldots, x_{n}\right\| \leq 1\right\}=0 .
$$

Now let $F, G \in L_{n}\left(V^{n}, W\right)$. Then for all $x_{1}^{n} \in V$, we have

$$
\begin{aligned}
\left\|(F \oplus G)\left(x_{1}^{n}\right)\right\| & =\left\|F\left(x_{1}^{n}\right)+G\left(x_{1}^{n}\right)\right\| \\
& \leq\left\|F\left(x_{1}^{n}\right)\right\|+\left\|G\left(x_{1}^{n}\right)\right\| \\
& \leq\|F\|\left\|x_{1}, \ldots, x_{n}\right\|+\|G\|\left\|x_{1}, \ldots, x_{n}\right\| \\
& =(\|F\|+\|G\|)\left\|x_{1}, \ldots, x_{n}\right\| .
\end{aligned}
$$

According to the definition of $\|\cdot\|$, it follows that $\|F \oplus G\| \leq\|F\|+\|G\|$. Also, for $\alpha \in \mathbb{R}$ and $x_{1}^{n} \in V$,

$$
\sup \left\|(\alpha \circ F)\left(x_{1}^{n}\right)\right\| \leq \sup \left\|\alpha \circ_{2} F\left(x_{1}^{n}\right)\right\| \leq|\alpha|\left\|F\left(x_{1}^{n}\right)\right\| \leq|\alpha|\|F\|\left\|x_{1}, \ldots, x_{n}\right\| .
$$

Now suppose that $\alpha \neq 0$. Hence,

$$
\|F\| \leq \sup \left\|\frac{1}{\alpha} \circ(\alpha \circ F)\right\| \leq \sup \left|\frac{1}{\alpha}\right|\|\alpha \circ F\|,
$$

and thus $|\alpha|\|F\| \leq \sup \|\alpha \circ F\|$. Then, $\sup \|\alpha \circ F\|=|\alpha|\|F\|$, for any $\alpha \neq 0$. Moreover, the equality is true for $\alpha=0$. This completes the proof.

Theorem 4.4. Let $\left(V,{ }_{1},{ }^{\circ}, \mathbb{R}\right)$ be an n-normed hypervector space and $\left(W,+_{2},{ }_{2},\|\|, \mathbb{R}\right)$ be a normed hypervector space. If $F: V^{n} \rightarrow W$ is a bounded n-homomorphism, then

(i) $\|F\| \leq M$, for any $M \in P^{(F)}$, where

$$
P^{(F)}=\left\{K>0: \forall x_{1}^{n} \in V,\left\|F\left(x_{1}^{n}\right)\right\| \leq K\left\|x_{1}, \ldots, x_{n}\right\|\right\} .
$$


(ii) $\left\|F\left(x_{1}^{n}\right)\right\| \leq\|F\|\left\|x_{1}, \ldots, x_{n}\right\|$, for all $x_{1}^{n} \in V$. Moreover,

$$
\begin{aligned}
\|F\| & =\sup \left\{\left\|F\left(x_{1}^{n}\right)\right\| ; x_{1}^{n} \in V,\left\|x_{1}, \ldots, x_{n}\right\| \leq 1\right\} \\
& =\sup \left\{\frac{\left\|F\left(x_{1}^{n}\right)\right\|}{\left\|x_{1}, \ldots, x_{n}\right\|} ; x_{1}^{n} \in V,\left\|x_{1}, \ldots, x_{n}\right\| \neq 0\right\} .
\end{aligned}
$$

Proof. The proof of (i) and first part of (ii) is clear. Hence, we prove the last part. Since $\left\|F\left(x_{1}^{n}\right)\right\| \leq\|F\|\left\|x_{1}, \ldots, x_{n}\right\|$, for all $x_{1}^{n} \in V$, we have $\frac{\left\|F\left(x_{1}^{n}\right)\right\|}{\left\|x_{1}, \ldots, x_{n}\right\|} \leq\|F\|$, when $\left\|x_{1}, \ldots, x_{n}\right\| \neq 0$. Therefore,

$$
\sup \left\{\frac{\left\|F\left(x_{1}^{n}\right)\right\|}{\left\|x_{1}, \ldots, x_{n}\right\|}: x_{1}^{n} \in V,\left\|x_{1}, \ldots, x_{n}\right\| \neq 0\right\} \leq\|F\| .
$$

Now set $\alpha=\sup \left\{\left\|F\left(x_{1}^{n}\right)\right\|: x_{1}^{n} \in V,\left\|x_{1}, \ldots, x_{n}\right\| \leq 1\right\}$. Then,

$$
\begin{aligned}
\alpha & \leq \sup \left\{\frac{\left\|F\left(x_{1}^{n}\right)\right\|}{\left\|x_{1}, \ldots, x_{n}\right\|}: x_{1}^{n} \in V,\left\|x_{1}, \ldots, x_{n}\right\| \leq 1\right\} \\
& \leq \sup \left\{\frac{\left\|F\left(x_{1}^{n}\right)\right\|}{\left\|x_{1}, \ldots, x_{n}\right\|}: x_{1}^{n} \in V,\left\|x_{1}, \ldots, x_{n}\right\| \neq 0\right\} \\
& \leq\|F\| .
\end{aligned}
$$

Now, let $x_{1}^{n} \in V$, such that $\left\|x_{1}, \ldots, x_{n}\right\| \neq 0$. Since

$$
\sup \left\|x_{1}^{i-1}, \frac{1}{\left\|x_{1}, \ldots, x_{n}\right\|} \circ_{1} x_{i}, x_{i+1}^{n}\right\|=\frac{1}{\left\|x_{1}, \ldots, x_{n}\right\|}\left\|x_{1}, \ldots, x_{n}\right\|=1 \text {, }
$$

for every $\left(y_{1}, \ldots, y_{n}\right) \in\left(x_{1}, \ldots, x_{i-1}, \frac{1}{\left\|x_{1}, \ldots, x_{n}\right\|}{ }^{\circ} 1 x_{i}, x_{i+1}, \ldots, x_{n}\right)$, we have $\left\|y_{1}, \ldots, y_{n}\right\| \leq 1$. Therefore, 


$$
\begin{aligned}
\frac{1}{\left\|x_{1}, \ldots, x_{n}\right\|}\left\|F\left(x_{1}^{n}\right)\right\| & =\sup \left\|\frac{1}{\left\|x_{1}, \ldots, x_{n}\right\|}{ }^{\circ} F\left(x_{1}^{n}\right)\right\| \\
& =\sup \left\|F\left(x_{1}^{i-1}, \frac{1}{\left\|x_{1}, \ldots, x_{n}\right\|}{ }^{\circ} x_{i}, x_{i+1}^{n}\right)\right\| \\
& \leq \alpha .
\end{aligned}
$$

On the other hand, if $x_{1}^{n} \in V$ and $\left\|x_{1}, \ldots, x_{n}\right\|=0$, then

$$
0 \leq\left\|F\left(x_{1}^{n}\right)\right\| \leq\|F\|\left\|x_{1}, \ldots, x_{n}\right\|=0
$$

and so $\left\|F\left(x_{1}^{n}\right)\right\|=0$. Thus, for all $x_{1}^{n} \in V$, we have

$$
\left\|F\left(x_{1}^{n}\right)\right\| \leq \alpha\left\|x_{1}, \ldots, x_{n}\right\|
$$

Then, $\alpha \in P^{(F)}$ and therefore $\|F\| \leq \alpha$ by (i). Hence, the proof is completed.

\section{Quasi-n-Norms on Hypervector Spaces}

In this section, the concepts of quasi- $n$-norm on product of hypervector spaces and quasi-n-normed subhyperspace are defined and some results about them are investigated.

Definition 5.1. Let $\left(V_{i},{ }_{i},{ }_{i}, \mathbb{R}\right)$ be a real hypervector space, for any $1 \leq i \leq n$. Then a function

$$
\|., \ldots, .\|: V_{1} \times \cdots \times V_{n} \rightarrow[0, \infty)
$$

is said to be a quasi-n-norm on $V_{1} \times \cdots \times V_{n}$ if for any $\left(x_{1}^{n}\right) \in V_{1} \times \cdots \times V_{n}, a \in \mathbb{R}$ and $1 \leq i \leq n$, the followings hold:

$(\mathrm{QnN} 1)\left\|x_{1}, \ldots, x_{n}\right\|=\left\|x_{\sigma(1)}, \ldots, x_{\sigma(n)}\right\|$, for all $\sigma \in \mathbb{S}_{n}$

$(\mathrm{QnN} 2)\left\|x_{1}, \ldots, x_{n-1}, y+z\right\| \leq\left\|x_{1}, \ldots, x_{n-1}, y\right\|+\left\|x_{1}, \ldots, x_{n-1}, z\right\|$;

$(\mathrm{QnN} 3) \sup \left\|x_{1}, \ldots, x_{n-1}, a \circ x_{n}\right\|=|a|\left\|x_{1}, \ldots, x_{n}\right\|$. 
In this case, the pair $\left(V_{1} \times \cdots \times V_{n},\|, \ldots\|,\right)$ is called a quasi- $n$ normed hypervector space.

Example 5.2. Assume $S$ as the set of all sequences of real numbers. For every $\left\{r_{k}\right\},\left\{s_{k}\right\} \in S$ and $\alpha \in \mathbb{R}$ define $\left\{r_{k}\right\}+\left\{s_{k}\right\}=\left\{r_{k}+s_{k}\right\}$ and $\alpha \circ\left\{s_{k}\right\}=\left\{\left\{t_{k}\right\}\right\}$, where $t_{k}$ is in the segment between 0 and $\alpha \cdot s_{k}$ for $k \in \mathbb{N}$. Then $(S,+, \circ, \mathbb{R})$ is a hypervector space. Now, for all $\left\{r_{1 k}\right\}, \ldots,\left\{r_{n_{k}}\right\} \in S$, let

$$
\left\|\left\{r_{1 k}\right\}, \ldots,\left\{r_{n_{k}}\right\}\right\|=\sum_{k=1}^{\infty}\left|r_{1 k}\right| \cdots\left|r_{n_{k}}\right|
$$

Set $\mathcal{B}=\left\{\left(\left\{r_{1 k}\right\}, \ldots,\left\{r_{n_{k}}\right\}\right) \in S^{n}:\left\|\left\{r_{1 k}\right\}, \ldots,\left\{r_{n_{k}}\right\}\right\|<\infty\right\}$. Then it can be seen that $\mathcal{B}$ is a quasi- $n$-normed subhyperspace of $S$.

Definition 5.3. Let $V=\left(V,+_{1}, \circ_{1},\|\cdot\|_{1}, \mathbb{R}\right)$ and $W=\left(W,+_{2}, \circ_{2}, \mathbb{R}\right)$ be real hypervector spaces and $\mathcal{W} \subseteq W^{n}$ be a quasi- $n$-normed subhyperspace. Then $\mathcal{M}_{\mathcal{W}} \subseteq L(V, W)^{n}$ is a set that $\left(f_{1}^{n}\right) \in \mathcal{M}_{\mathcal{W}}$, where for every $x \in V$, the following conditions hold:

(i) $\left(f_{1}^{n}(x)\right) \in \mathcal{W}$;

(ii) There exists $M>0$ such that $\left\|f_{1}^{n}(x)\right\| \leq M\|x\|_{1}^{n}$.

Lemma 5.4. Let $V=\left(V,+_{1},{ }_{1},\|\cdot\|_{1}, \mathbb{R}\right)$ and $W=\left(W,+_{2},{ }^{\circ}, \mathbb{R}\right)$ be hypervector spaces. Then $\mathcal{M}_{\mathcal{W}}\left(g_{2}^{n}\right)=\left\{h \in L(V, W):\left(h, g_{2}^{n}\right) \in \mathcal{M}_{\mathcal{W}}\right\}$ and $\left(g_{1}^{n-1}\right) \mathcal{M}_{\mathcal{W}}=\left\{h \in L(V, W):\left(g_{1}^{n-1}, h\right) \in \mathcal{M}_{\mathcal{W}}\right\}$ are subhyperspaces of the $L(V, W)$, for every $g_{1}^{n} \in L(V, W)$.

Proof. Let $g_{2}^{n}: V \rightarrow W$ be strong homomorphism. Assume that $h: V \rightarrow W$ is a strong homomorphism such that $h(x)=0$ for all $x \in V$. For every subhyperspace $H$ of $W$, we have $0 \in H$. Since $\mathcal{W}^{g_{2}^{n}(x)}$ is a 
subhyperspace of $W$ for all $x \in V$, then $h(x)=0 \in \mathcal{W}^{g_{2}^{n}(x)}$. Thus, $\left(h(x), g_{2}^{n}(x)\right) \in \mathcal{W}$ for all $x \in V$. Also, if $M>0$, then

$$
\left\|h(x), g_{2}^{n}(x)\right\|=\left\|0, g_{2}^{n}(x)\right\|=0 \leq M\|x\|_{1}^{n} .
$$

So $\left(h, g_{2}^{n}\right) \in \mathcal{M}_{\mathcal{W}}$ and therefore $h \in \mathcal{M}_{\mathcal{W}}\left(g_{2}^{n}\right)$. Then $\mathcal{M}_{\mathcal{W}}\left(g_{2}^{n}\right) \neq \emptyset$. Now, let $f_{1}, f_{2} \in \mathcal{M}_{\mathcal{W}}\left(g_{2}^{n}\right)$. Thus, $\left(f_{1}(x), g_{2}^{n}(x)\right),\left(f_{1}(x), g_{2}^{n}(x)\right) \in \mathcal{W}$ and so $f_{1}(x), f_{2}(x) \in \mathcal{W}^{g_{2}^{n}(x)}$. Since $\mathcal{W}^{g_{2}^{n}(x)}$ is a subhyperspace of $W$, then $f_{1}+f_{2} \in \mathcal{W}^{g_{2}^{n}(x)}$ and we have $\left(\left(f_{1}+f_{2}\right)(x), g_{2}^{n}(x)\right) \in \mathcal{W}$ for every $x \in V$. Moreover, there exist $M_{1}, M_{2}>0$ such that $\left\|f_{1}(x), g_{2}^{n}(x)\right\| \leq M_{1}\|x\|_{1}^{n}$ and $\left\|f_{2}(x), g_{2}^{n}(x)\right\| \leq M_{2}\|x\|_{1}^{n}$, for every $x \in V$, Hence,

$$
\left\|f_{1}(x)+f_{2}(x), g_{2}^{n}(x)\right\| \leq\left\|f_{1}(x), g_{2}^{n}(x)\right\|+\left\|f_{2}(x), g_{2}^{n}(x)\right\| \leq\left(M_{1}+M_{2}\right)\|x\|_{1}^{n} .
$$

Therefore, $\left(f_{1}+f_{2}, g_{2}^{n}\right) \in \mathcal{M}_{\mathcal{W}}$ and so $f_{1}+f_{2} \in \mathcal{M}_{\mathcal{W}}\left(g_{2}^{n}\right)$. Now, suppose that $\alpha \in \mathbb{R}$ and $f \in \mathcal{M}_{\mathcal{W}}\left(g_{2}^{n}\right)$. It follows that $\left(f(x), g_{2}^{n}(x)\right) \in \mathcal{W}$ and consequently, $f(x) \in \mathcal{W}^{g_{2}^{n}(x)}$, for every $x \in V$. Since, $\mathcal{W}^{g_{2}^{n}(x)}$ is a subhyperspace, then $\alpha \circ_{2} f(x) \subseteq \mathcal{W}^{g_{2}^{n}(x)}$ and so

$$
\left(\alpha \circ_{2} f(x), g_{2}^{n}(x)\right)=\left\{\left(t, g_{2}^{n}(x)\right): t \in \alpha \circ_{2} f(x)=f\left(\alpha \circ_{1} x\right)\right\} \subseteq \mathcal{W} .
$$

Moreover, there exists $M>0$ such that $\left\|f(x), g_{2}^{n}(x)\right\| \leq M\|x\|_{1}^{n}$. Hence, for all $h \in \alpha_{\circ_{2}} f$, we have

$$
\left\|h(x), g_{2}^{n}(x)\right\| \leq \sup \left\|\alpha \circ_{2} f(x), g_{2}^{n}(x)\right\|=|\alpha| \cdot\left\|f(x), g_{2}^{n}(x)\right\| \leq|\alpha| \cdot M\|x\|_{1}^{n},
$$

for every $x \in V$. Therefore $\left(h, g_{2}^{n}\right) \in \mathcal{M}_{\mathcal{W}}$ and so $h \in \mathcal{M}_{\mathcal{W}}\left(g_{2}^{n}\right)$, for all $h \in \alpha \circ_{2} f$. Hence $\alpha \circ_{2} f \subseteq \mathcal{M}_{\mathcal{W}}\left(g_{2}^{n}\right)$. So $\mathcal{M}_{\mathcal{W}}\left(g_{2}^{n}\right)$ is a subhyperspace of $L(V, W)$. Similarly, it can be shown that $\left(g_{1}^{n-1}\right) \mathcal{M}_{\mathcal{W}}$ is a subhyperspace of $L(V, W)$. 
Theorem 5.5. Let $V=\left(V,+_{1},{ }_{1},\|\cdot\|_{1}, \mathbb{R}\right)$ and $W=\left(W,+_{2}, \circ_{2}, \mathbb{R}\right)$ be hypervector spaces and $\left(f_{1}^{n}\right) \in \mathcal{M}_{\mathcal{W}}$, such that $\mathcal{M} \subseteq W^{n}$, define

$$
\left\|f_{1}^{n}\right\|=\inf \left\{M>0: \forall x \in V,\left\|f_{1}^{n}(x)\right\| \leq M\|x\|_{1}^{n}\right\} .
$$

Then,

(i) $\left\|f_{1}^{n}\right\| \leq M$, for every $M \in P^{\left(f_{1}^{n}\right)}$, where

$$
P^{\left(f_{1}^{n}\right)}=\left\{K>0: \forall x \in V,\left\|f_{1}^{n}(x)\right\| \leq K\|x\|_{1}^{n}\right\} .
$$

(ii) $\left\|f_{1}^{n}(x)\right\| \leq\left\|f_{1}^{n}\right\|\|x\|_{1}^{n}$, for all $x \in V$.

(iii) $\left\|f_{1}^{n}\right\|=\sup \left\{\left\|f_{1}^{n}(x)\right\|: x \in V,\|x\|_{1} \leq 1\right\}=\sup \left\{\frac{\left\|f_{1}^{n}(x)\right\|}{\|x\|_{1}^{n}}: x \in V,\|x\|_{1} \neq 0\right\}$.

(iv) If $\mathcal{W}$ is a quasi-n-normed subhyperspace, then $\left\|f_{1}^{n}\right\|=\left\|f_{\delta(1)}^{\delta(n)}\right\|$, for every $\left(f_{1}^{n}\right) \in \mathcal{M}_{\mathcal{W}}$.

Proof. The proofs of (i), (ii) and (iv) are clear. We prove (iii). By (ii), we have

$$
\sup \left\{\left\|f_{1}^{n}(x)\right\|: x \in V,\|x\|_{1}\right\} \leq\left\|f_{1}^{n}\right\| .
$$

Let $\alpha=\sup \left\{\left\|f_{1}^{n}(x)\right\|: x \in V,\|x\|_{1}\right\}$ and $x \in V$, with $x \neq 0$. Then,

$$
\begin{aligned}
\left\|f_{1}^{n}(x)\right\| & =\sup \left\|\left(\frac{1}{\|x\|_{1}}\|x\|_{1}\right) \circ_{2} f_{1}(x), \ldots,\left(\frac{1}{\|x\|_{1}} \cdot\|x\|_{1}\right) \circ_{2} f_{n}(x)\right\| \\
& =\sup \|\| x\left\|_{1} \circ_{2} f_{1}\left(\frac{1}{\|x\|_{1}} \circ_{1} x\right), \ldots,\right\| x\left\|_{1} \circ_{2} f_{n}\left(\frac{1}{\|x\|_{1}} \circ_{1} x\right)\right\| \\
& =\|x\|_{1}^{n} \sup \left\|f_{1}\left(\frac{1}{\|x\|_{1}} \circ_{1} x\right), \ldots, f_{n}\left(\frac{1}{\|x\|_{1}} \circ_{1} x\right)\right\| .
\end{aligned}
$$


If $y \in \frac{1}{\|x\|_{1}} \circ_{1} x$, then $\|y\|_{1} \leq \sup \left\|\frac{1}{\|x\|_{1}} \circ_{1} x\right\|=\frac{1}{\|x\|_{1}}\left\|x_{1}\right\|=1$ and $\left\|f_{1}^{n}(y)\right\| \leq \alpha$.

Therefore, $\left\|f_{1}^{n}(x)\right\| \leq \alpha\|x\|_{1}^{n}$, for $\quad x \neq 0$. Also, for $x=0$, we have $\left\|f_{1}^{n}(x)\right\|=0 \leq \alpha\|x\|_{1}^{n}$. Hence, $\left\|f_{1}^{n}(x)\right\| \leq \alpha\|x\|_{1}^{n}$, for every $x \in V$. This implies that $\alpha \in P^{\left(f_{1}^{n}\right)}$. By (i), we have $\left\|f_{1}^{n}\right\| \leq \alpha$ and by (1) we conclude that $\left\|f_{1}^{n}\right\|=\sup \left\{\left\|f_{1}^{n}(x)\right\|: x \in V,\|x\|_{1} \leq 1\right\}$. Now, suppose that $0 \neq x \in V$. By (ii), we have

$$
\frac{\left\|f_{1}^{n}(x)\right\|}{\|x\|_{1}^{n}} \leq\left\|f_{1}^{n}\right\|
$$

and $\quad$ so $\quad \sup \left\{\frac{\left\|f_{1}^{n}(x)\right\|}{\|x\|_{1}^{n}}: x \in V,\left\|x_{1}\right\| \neq 0\right\} \leq\left\|f_{1}^{n}\right\|$. Thus, if $\quad$ we $\quad$ put $\beta=\sup \left\{\frac{\left\|f_{1}^{n}(x)\right\|}{\|x\|_{1}^{n}}: x \in V,\left\|x_{1}\right\| \neq 0\right\}$, then $\left\|f_{1}^{n}(x)\right\| \leq \beta\|x\|_{1}^{n}$, for every $x \neq 0$.

On the other hand, if $x=0$, then $\left\|f_{1}^{n}\right\|=0$. Thus $\left\|f_{1}^{n}(x)\right\| \leq \beta\|x\|_{1}^{n}$, for every $x \in V$, which means that $\beta \in P^{\left(f_{1}^{n}\right)}$ and $\left\|f_{1}^{n}\right\| \leq \beta$. This completes the proof.

Theorem 5.6. Let $V=\left(V,+_{1}, \circ_{1},\|\cdot\|_{1}, \mathbb{R}\right)$ and $W=\left(W,+_{2}, \circ_{2}, \mathbb{R}\right)$ be hypervector spaces and $\mathcal{W}$ be a quasi-n-normed subhyperspace. Then $\mathcal{M}_{\mathcal{W}}$ is an quasi-n-normed subhyperspace with the following $n$-norm:

$$
\left\|f_{1}^{n}\right\|=\sup \left\{\left\|f_{1}^{n}(x)\right\|: x \in V,\|x\|_{1} \leq 1\right\} .
$$

Proof. Let $\left(f_{1}^{n}\right) \in \mathcal{M}_{\mathcal{W}}$. It means that there exists $M>0$ such that $\left\|f_{1}^{n}\right\| \leq M\|x\|_{1}^{n}$, for every $x \in V$. Therefore,

$$
\sup \left\{\left\|f_{1}^{n}(x)\right\|: x \in V,\|x\|_{1} \leq 1\right\} \leq M<\infty,
$$

and so $\|., \ldots,$.$\| has finite nonnegative values. Moreover, we have the$ following: 
(i) If $x \in V,\|x\|_{1} \leq 1$ and $\alpha \in \mathbb{R}$, then

$$
\begin{aligned}
\sup \left\|f_{1}^{i-1}(x),\left(\alpha \circ_{1} f_{i}\right)(x), f_{i+1}^{n}(x)\right\| & \leq \sup \left\|f_{1}^{i-1}(x), \alpha \circ_{2} f_{i}(x), f_{i+1}^{n}(x)\right\| \\
& =|\alpha| \cdot\left\|f_{1}^{n}(x)\right\| \\
& \leq|\alpha| \cdot \sup \left\{\left\|f_{1}^{n}(x)\right\|: x \in V,\|x\|_{1} \leq 1\right\} \\
& =|\alpha| \cdot\left\|f_{1}^{n}\right\|,
\end{aligned}
$$

and consequently, we have

(2) $\sup \left\|f_{1}^{i-1}, \alpha \circ_{1} f_{i}, f_{i+1}^{n}\right\| \leq|\alpha| \cdot\left\|f_{1}^{n}\right\|$.

Now, let $\alpha \neq 0$. Using (2), we have

$$
\left\|f_{1}^{n}\right\| \leq \sup \left\|f_{1}^{i-1},\left(\frac{1}{\alpha} \alpha\right) \circ_{1} f_{i}, f_{i+1}^{n}\right\| \leq \frac{1}{\alpha} \sup \left\|f_{1}^{i-1}, \alpha \circ_{1} f_{i}, f_{i+1}^{n}\right\|,
$$

and $\quad|\alpha|\left\|f_{1}^{n}\right\| \leq \sup \left\|f_{1}^{i-1}(x), \alpha \circ_{1} f_{i}, f_{i+1}^{n}\right\|, \quad$ for $\quad \alpha \neq 0 . \quad$ On $\quad$ the other hand, if $\alpha=0$, then $|\alpha|\left\|f_{1}^{n}\right\|=0=\sup \left\|f_{1}^{i-1}, \alpha \circ_{1} f_{i}, f_{i+1}^{n}\right\|$. So $|\alpha|\left\|f_{1}^{n}\right\| \leq \sup \left\|f_{1}^{i-1}, \alpha \circ_{1} f_{i}, f_{i+1}^{n}\right\|$, for all $\alpha \in \mathbb{R}$, and by using (2), we have $\sup \left\|f_{1}^{i-1}, \alpha \circ_{1} f_{i}, f_{i+1}^{n}\right\|=|\alpha| \cdot\left\|f_{1}^{n}\right\|$.

(ii) Suppose that $f_{1}^{i-1}, g, h, f_{i+1}^{n} \in L(V, W)$, such that $\left(f_{1}^{i-1}, g, f_{i+1}^{n}\right)$, $\left(f_{1}^{i-1}, h, f_{i+1}^{n}\right) \in \mathcal{M}_{\mathcal{W}}$, for $1 \leq i \leq n$. Let $x \in V$, such that $\|x\|_{1} \leq 1$. Then, we have

$$
\begin{aligned}
\left\|f_{1}^{i-1}(x),(g+h)(x), f_{i+1}^{n}(x)\right\|= & \left\|f_{1}^{i-1}(x), g(x)+h(x), f_{i+1}^{n}(x)\right\| \\
\leq & \left\|f_{1}^{i-1}(x), g(x), f_{i+1}^{n}(x)\right\|+\left\|f_{1}^{i-1}, h(x), f_{i+1}^{n}(x)\right\| \\
\leq & \sup \left\{\left\|f_{1}^{i-1}(x), g(x), f_{i+1}^{n}(x)\right\|: x \in V,\|x\|_{1} \leq 1\right\} \\
& +\sup \left\{\left\|f_{1}^{i-1}(x), h(x), f_{i+1}^{n}(x)\right\|: x \in V,\|x\|_{1} \leq 1\right\} \\
= & \left\|f_{1}^{i-1}, g, f_{i+1}^{n}\right\|+\left\|f_{1}^{i-1}, h, f_{i+1}^{n}\right\| .
\end{aligned}
$$


It follow that, $\left\|f_{1}^{i-1}, g+h, f_{i+1}^{n}\right\| \leq\left\|f_{1}^{i-1}, g, f_{i+1}^{n}\right\|+\left\|f_{1}^{i-1}, h, f_{i+1}^{n}\right\|$. Now since $\mathcal{W}$ is quasi- $n$-normed, so $\mathcal{M}_{\mathcal{W}}=\mathcal{M}_{\mathcal{W}}^{-1}$ and we have

$$
\begin{aligned}
\left\|f_{1}^{n}\right\| & =\sup \left\{\left\|f_{1}^{n}(x)\right\|: x \in V,\|x\|_{1} \leq 1\right\} \\
& =\sup \left\{\left\|f_{\sigma(1)}^{\sigma(n)}(x)\right\|: x \in V,\|x\|_{1} \leq 1\right\} \\
& =\left\|f_{\sigma(1)}^{\sigma(n)}\right\|,
\end{aligned}
$$

for all $\sigma \in \mathbb{S}_{n}$. Moreover, $\mathcal{M}_{\mathcal{W}}\left(g_{2}^{n}\right)$ and $\left(g_{1}^{n-1}\right) \mathcal{M}_{\mathcal{W}}$ are subhyperspaces of $L(V, W)$, for all $g_{1}^{n} \in L(V, W)$, by Lemma 5.4. Therefore, $\mathcal{M}_{\mathcal{W}}$ is quasi- $n$-normed subhyperspace.

\section{References}

[1] A. A. A. Agboola and S. A. Akinleye, On neutrosophic hypervector spaces, ROMAI J. 11(1) (2015), 1-16.

[2] R. Ameri and O. R. Dehghan, On dimension of hypervector spaces, Eur. J. Pure Appl. Math. 1(2) (2008), 32-50.

[3] P. Corsini, Prolegomena of Hypergroup Theory, Second Edition, Aviani Editor, 1993.

[4] P. Corsini and V. Leoreanu, Applications of Hyperstructure Theory, Kluwer Academic Publications, 2003.

[5] B. Davvaz and V. Leareanu-Fotea, Hyperring Theory and Applications, International Academic Press, USA, 2007.

[6] S. Gähler, Linear 2-normierte Rume, Math. Nachr. 28 (1964), 1-43.

[7] F. Marty, Sur une generalization de la notion de groupe, 8th congress des Mathematiciens Scandinaves, Stockholm (1934), 45-49.

[8] A. Misiak, $n$-inner product spaces, Math. Nachr. 140 (1989), 299-319.

[9] P. Raja and S. M. Vaezpour, Normed hypervector spaces, Iran. J. Math. Sci. Inform. 2(2) (2007), 35-44.

[10] M. S. Tallini, Hypervector spaces, 4th Int. Congress on AHA (1990), 167-174.

[11] M. S. Tallini, Weak hypervector spaces and norms in such spaces, Algebraic Hyperstructures and Applications, Hadronic Press (1994), 109-206. 
[12] M. S. Tallini, Characterization of remarkable hypervector spaces, Proc. 8th Int. Congress on AHA, Samotraki, Greece (2002), 231-237.

[13] T. Vougiuklis, Hyperstructures and their Representations, Hardonic Press Inc., 1994.

[14] T. Vougiuklis, $H_{v}$-vector spaces, Proc. 5th Int. Congress on AHA, Jasi, Rumania (1994), 181-190. 